\section{Deleted in Liver Cancer 2 (DLC2) protein expression in hepatocellular carcinoma}

\author{
Dominika Wolosz, ${ }^{1}$ \\ Agnieszka Walczak, 2,3 \\ Grzegorz Szparecki, ${ }^{1}$ Michal Dwojak, ${ }^{4,5}$ \\ Magdalena Winiarska, ${ }^{4}$ Ewa Wolinska, ${ }^{1}$ \\ Barbara Gornicka ${ }^{1}$ \\ ${ }^{1}$ Department of Pathology, Medical \\ University of Warsaw \\ ${ }^{2}$ Laboratory of Molecular and Systemic \\ Neuromorphology, Nencki Institute of \\ Experimental Biology, Polish Academy \\ of Sciences, Warsaw \\ ${ }^{3}$ Department of Gene Expression, \\ Institute of Molecular Biology and \\ Biotechnology, Adam Mickiewicz \\ University, Poznan \\ ${ }^{4}$ Department of Immunology, Center for \\ Biostructure Research, Medical \\ University of Warsaw \\ ${ }^{5}$ Postgraduate School of Molecular \\ Medicine, Warsaw, Poland
}

\begin{abstract}
Deleted in Liver Cancer (DLC) proteins belong to the family of RhoGAPs and are believed to operate as negative regulators of the Rho family of small GTPases. So far, the role of the first identified member from the DLC family, DLC1, was established as a tumor suppressor in hepatocellular carcinoma. The function of its close family relative, DLC2 is unequivocal. In the present study we attempted to determine whether the loss of DLC2 is a common feature of hepatocellular carcinoma tissue. We examined two types of hepatocellular carcinomatypical and fibrolamellar one. Our analysis revealed that DLC2 protein is not diminished in cancer tissue when compared to non-cancerous liver specimens. What is more, we observed DLC2 to be more abundantly expressed in cancer tissue, particularly in tumors with the inflammation background. In addition, we found that $D L C 2$ gene status was diploid in virtually all tumor samples examined. Our results indicate that DLC2 is not diminished in hepatocellular carcinoma cells. It appears that members of the DLC family, although structurally highly related, may function differently in cancer cells.
\end{abstract}

\section{Introduction}

Hepatocellular carcinoma (HCC) remains a serious oncological issue and represents the fourth leading cause of cancerrelated deaths worldwide. ${ }^{1,2}$ This is the most common primary liver cancer which develops predominantly in patients with underlying cirrhotic liver disease of various etiologies. $^{3}$ Although great progress has been made in understanding the biology of cancer in recent decades, the pathophysiology of HCC is still poorly characterized. As many studies highlight $\mathrm{HCC}$ is a complex disease and there is no dominant molecular pathway which underlies its development and progression. ${ }^{4}$ To date, tumor resection seems the most effective method of treatment; alternatively, in some cases of liver transplantation, chemoembolization and thermoablation may be also beneficial for patients. ${ }^{5}$ Within the group of hepatocellular carcinomas, the fibrolamellar variant (FL) is particularly interesting as its etiology is completely different. It usually affects young adults and most patients do not have liver cirrhosis or other co-existing liver disease. ${ }^{6}$ To date there are not many literature data available describing the risk factors and molecular changes associated with the development of FL. ${ }^{7-9}$

Findings of numerous studies showed that Rho signaling pathways are altered in human cancers. ${ }^{10}$ One of the major causes of these alterations is the disruption of genes encoding proteins that regulate Rho proteins function, namely Rho GTPase activating proteins (RhoGAPs). DLC2 is a member of broader Deleted in Liver Cancer protein family, which belongs to the RhoGAP super family. ${ }^{11}$ Typical DLC protein contains three functional domains - the Sterile Alpha Motif (SAM), the Rho GAP and Steroidogenic Acute Regulatory Related Lipid Transfer (START) domain. ${ }^{12}$ The tumor suppressor function is linked mainly to its RhoGAP activity, however other parts of the protein, i.e., SAM domain may regulate this activity as well. ${ }^{13-15}$

The best characterized member of DLC family, $D L C 1$, is frequently deleted in different types of cancer and its tumor suppressive function has been clearly evidenced. ${ }^{16,17}$ The following identified gene from the DLC family, $D L C 2$, has been studied to a lesser extent. This gene is located on chromosome $13 \mathrm{q} 12$, a region exposed to the allelic loss, as was documented in several human cancers. ${ }^{18}$ The DLC2 protein is $50 \%$ identical to DLC1, shares the same functional domains and functions through a similar mode of action. DLC2 expression was found to be down-regulated in several types of cancer, including lung, renal, ovarian,
Corresponding Author: Ewa Wolinska, Department of Pathology, Medical University of Warsaw, Żwirki i Wigury 61, 02-091 Warsaw, Poland.

Tel. +48.22.5991682 - Fax: +48.22.5991671. E-mail: ewa.wolinska7@gmail.com

Key words: DLC2; hepatocellular carcinoma; fibrolamellar hepatocellular carcinoma.

Contributions: DW, AW, GS, MD, MW, data collecting and analyzing; EW, data analyzing and manuscript writing; $\mathrm{BG}$, manuscript reviewing.

Conflict of interest: The authors declare no potential conflict of interest.

Funding: The present work was supported by the grant no. 3509/B/P01/2010/38 from the Polish State Committee for Scientific Research and by the Medical University of Warsaw, funding no. 1M11/PM11.

Received for publication: 12 September 2018. Accepted for publication: 8 February 2019.

This work is licensed under a Creative Commons Attribution-NonCommercial 4.0 International License (CC BY-NC 4.0).

CCopyright D. Wolosz et al., 2019

Licensee PAGEPress, Italy

European Journal of Histochemistry 2019; 63:2981 doi:10.4081/ejh.2019.2981

breast, colon and rectal tumors. ${ }^{19}$ To date, there are limited literature reports which address the DLC2 function, nevertheless, it has been shown that it can regulate the growth of tumor cells by modulating RhoA activity and thus inhibits cell motility. ${ }^{20}$ In addition, DLC2 was found to affect Ras signaling and inhibit Ras-mediated cell transformation. ${ }^{21}$

In this study we determined the presence and tissue/cellular localization of DLC2 protein in HCC tumor samples. To date, no studies have been carried out addressing the expression of DLC2 in the fibrolamellar subtype of HCC. Additionally, we designed the genetic probe for $D L C 2$ in order to find (by the in situ hybridization approach) whether the loss of this gene is a common feature in both typical and fibrolamellar variant of HCC.

\section{Materials and Methods}

\section{Tissue specimens}

Tumor samples were obtained from 81 patients with diagnosed HCC, among this group there were 9 tumors of the fibrolamellar subtype. Control group consisted 
of 25 normal liver tissue specimens. Additionally, 10 tissue samples with macroregenerative nodules were examined.

\section{Ethics statement}

The study was approved by the Medical University of Warsaw Ethics Committee $(\mathrm{KBO} / 42 / 11)$.

\section{Immunohistochemistry/ immunofluo- rescence}

The immunohistochemical staining, in brief, was as follows. Formalin-fixed, paraffin-embedded $4 \mu \mathrm{m}$ sections were deparaffinized and rehydrated. To unmask antigen sites sections were treated with high temperature boiling in the $0.01 \mathrm{~mol} / \mathrm{L}$ citrate buffer pH 6.0 (DakoCytomation, Glostrup, Denmark) for $2 \times 7 \mathrm{~min}$ in the microwave oven. Subsequently sections were incubated with $3 \%$ hydrogen peroxide to block endogenous peroxidase for $30 \mathrm{~min}$ and then treated with 5\% normal donkey serum (Jackson Immunoresearch, West Grope, PA, USA) for $30 \mathrm{~min}$ followed by Serum Free Protein Block (DakoCytomation) $10 \mathrm{~min}$ incubation to prevent unspecific binding of antibodies. Then the goat anti-DLC2 (Santa Cruz Biotechnology, Santa Cruz, CA, USA) antibody in 1:50 dilution was applied and incubated overnight in moist chamber in $4^{\circ} \mathrm{C}$. Detection of the primary antibody was performed with the donkey anti-goat peroxidase-conjugated (Jackson Immunoresearch) antibody in 1:500 dilution for $1 \mathrm{~h}$. To visualize the immunostaining 3, 3'diaminobenzidine (Dako) was used as a chromogen.

Immunohistochemical results of DLC2 staining were quantified by the morphometric analysis using a Nikon Eclipse 80i microscope and Image Pro Plus software. From each patient, 10 random fields were photographed at 20x magnification. On every image the area covered by the immunoreactivity, as well as the mean intensity of staining, were quantified. Within a given field, the product of immunoreaction intensity times the area was considered as an approximation of the total immunoreactivity, and displayed in arbitrary units. For immunofluorescence studies primary antibodies were detected with donkey anti- goat Alexa555 in dilution 1:200 (for DLC2) and donkey anti-rabbit Alexa488 in dilution 1:200 (for mitochondria) secondary antibodies conjugated with fluorophores (both from Invitrogen, Carlsbad, CA, USA). For colocalisation studies rabbit anti-hepatocytes antibody (Biogenex, Fremont, CA, USA) was applied. After immunostaining sections were mounted with the Vectashield mounting medium containing DAPI (Vector
Laboratories, Burlingame, CA, USA). Immunofluorescence was analyzed under a high-resolution Leica TCSSP5 Confocal microscope (Leica Microsystems, Wetzlar, Germany).

\section{Fluorescence in situ hybridization}

\section{Probe preparation}

The DLC2 sequence was obtained from BAC DNA library (CHORI, Children's Hospital Oakland Research Institute, Oakland, CA, USA) as bacterial LB agar stab culture. E. coli were cultured in LB agar with chloramphenicol, passaged as a single isolated colony and subjected to a rapid alkaline DNA isolation by EndoFree Plasmid Maxi Kit (Qiagen, Hilden, Germany). Extracted DNA was amplified by GenomiPhi DNA Amplification Kit (GE Healthcare, Chicago, IL, USA). Subsequently dUTP were labeled with digoxygenin or biotin Translation Mix (Roche Applied Science, USA) and probe was labeled by the nick-translation method according to Cremer et al. ${ }^{22}$

\section{Hybridization procedure - modified method of Cremer et al.}

For hybridization procedure sections were deparaffinized and hydrated in a series of alcohol. Subsequently digestion with pepsin and tissue permeabilization with sodium thiocyanate at $80^{\circ} \mathrm{C}$ were performed. Then sections were equilibrated in $50 \%$ formamide $/ 2 \mathrm{xSSC}$, prehybridized in $45^{\circ} \mathrm{C}$, denatured in $80^{\circ} \mathrm{C}$ and hybridized for $40 \mathrm{~h}$. In brief, post-hybridization procedures were done by incubation sections in $2 \mathrm{xSSC}$ and $0.1 \times S S C$ in $60^{\circ} \mathrm{C} 2 \times 10 \mathrm{~min}$. Detection of biotin-conjugated probes was performed by the incubation with avidin-Alexa488 (Molecular Probes, Eugene, OR, USA) and anti-avidin-FITC (Sigma Aldrich, St. Louis, MO, USA) antibodies. Detection of digoxygenin-conjugated probes was performed with the anti-digoxigenin - rhodamine sheep antibody (Roche Applied Science, Penzberg, Germany). In order to visualize the nuclei, sections were stained with Hoechst reagent (Molecular Probes). Specimens were examined under Nikon Eclipse 80i fluorescent microscope (Nikon, Tokyo, Japan). To assess the gene status in each section, 200 cells were counted at 600x magnification.

\section{Statistical analysis}

Differences between groups were analyzed by the non-parametric Mann-Whitney $U$ test. Statistical correlations were evaluated by the Spearman's rank correlation coefficient test.

\section{Results}

DLC2 immunoreactivity is more prominent in hepatocellular carcinoma when compared to the normal liver

In the normal liver DLC2 immunoreactivity was present in virtually all hepatocytes in a form of diffused, cytoplasmic staining (Figure 1a). Similar pattern of staining was observed in sections with macroregenerative nodules (Figure 1b). We found cancer cells to be more intensively stained when compared to normal hepatocytes (Figure $1 \mathrm{c}, \mathrm{d}$ ). What is more, in HCC cells, apart from cytoplasmic, nuclear DLC2 immunoreactivity was observed as well (Figure 1e). Statistical analysis of morphometric measures revealed significantly more DLC2 expression in HCC when compared to either normal liver or macroregenerative nodules (Mann-Whitney $\mathrm{U}$ test, $\mathrm{P}=$ 0.0004 and $\mathrm{P}=0.0034$ respectively, Figure 1 h). We found that DLC2 was more prominently expressed in these tumors that developed in the liver with the inflammation $(\mathrm{P}=0.049$; Mann-Whitney $U$ test; Figure 1 f,i). In FL sections the DLC2 staining pattern was similar to that observed in a typical HCC (Figure 1e). A negative control, performed with the use of rabbit immunoglobulins applied with the same concentration as the primary antibody, displayed no immunoreactivity (Figure $1 \mathrm{~g}$ ). To check whether DLC2 localizes to mitochondria we performed double immunofluorescence staining with anti-DLC2 and anti-mitochondria antibodies which was analyzed under the high-resolution confocal microscopy. We found that both DLC2 and mitochondria were more prominently expressed in tumor cells when compared to the adjacent liver (Figure $1 \mathrm{j}, \mathrm{k}$ ). However, we did not find profound overlap between these two immunoreactivities (Figure 1 1,m).

\section{The copy number of the DLC2 gene is diploid in $\mathrm{HCC}$ specimens}

Fluorescent in situ studies with probes designed for the $D L C 2$ gene showed that in the majority of tumor samples that were tested, the number of $D L C 2$ copies was diploid in cancer cells (Figure $2 \mathrm{a}, \mathrm{b}$ ). The loss of one copy of the $D L C 2$ gene was detected in 3 out of 18 examined tumors. In two of these cases the loss correlated with a slightly decreased DLC2 expression, but in one of them had no effect on the level of protein. The gene deletion was found to be independent of the HCC subtype. 

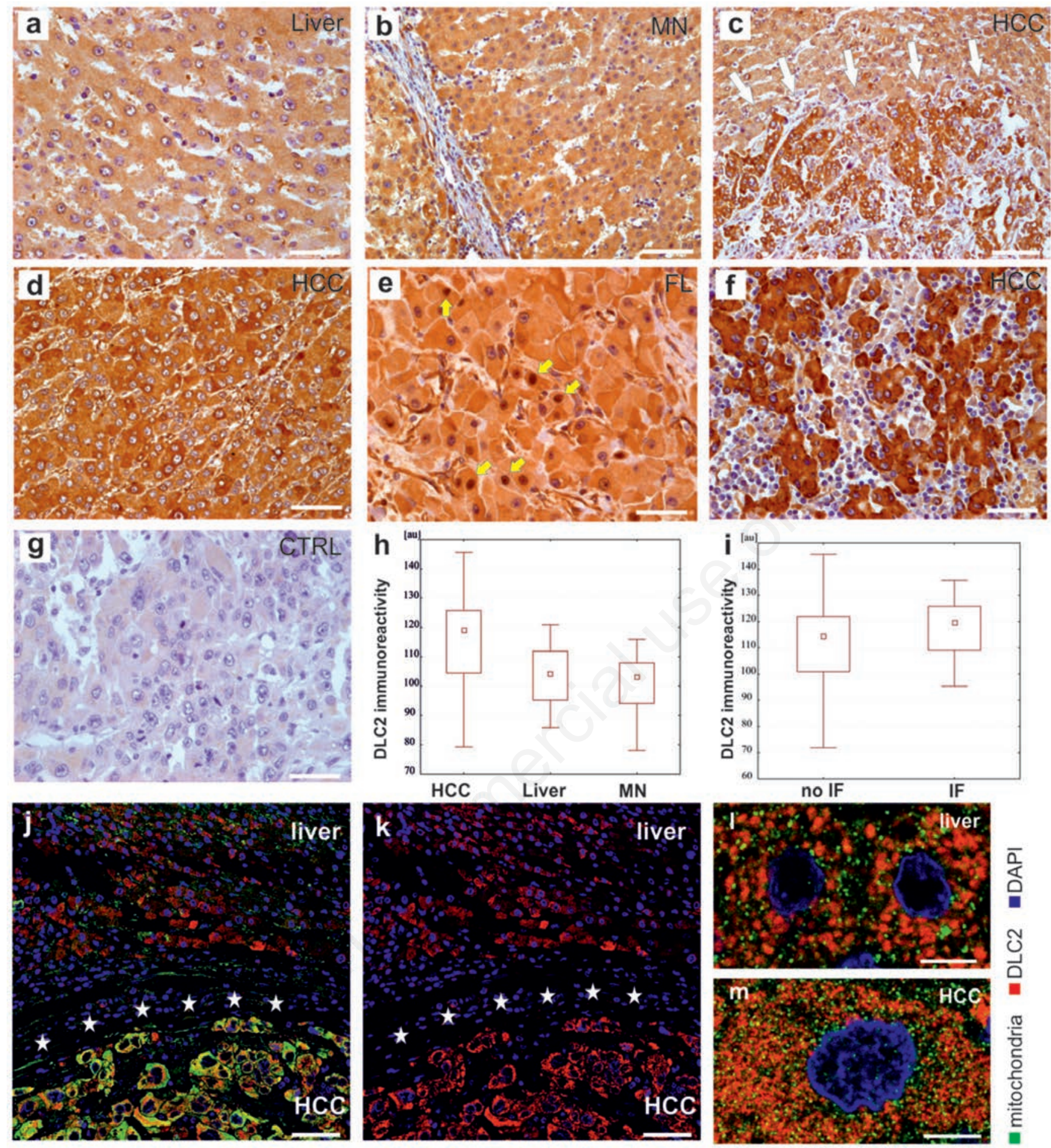

Figure 1. DLC2 immunoreactivity in the normal liver and hepatocellular carcinoma. a) Staining in the normal liver. b) Staining in macroregenerative nodules. c) DLC2 immunoreactivity on the border zone (marked with white arrows) between the normal liver and HCC. d) HCC with heterogeneous staining. e) Staining in FL, note the nuclear staining marked with yellow arrows. f) Strong staining in HCC with the inflammation. g) Control immunoreaction. h) A graph showing the difference in DLC2 immunoreactivity between normal liver, HCC and macroregenerative nodules, [au] arbitrary units. i) A graph showing the difference in DLC2 immunoreactivity between HCC with (IF) and without (no IF) inflammation. j) Combined immunofluorescence of DLC2 (red), mitochondria (green) and cell nuclei (blue); upper part of the image depicts the remaining liver, whereas lower part tumor masses (the border zone is pointed by white stars), note stronger both DLC2 and mitochondria immunoreactivities within cancer cells. $\mathrm{k}$ ) The same immunofluorescence image as in J with the extracted DLC2 immunofluorescence (red). 1, m) High power images with immunofluorescence of DLC2 (red) and mitochondria (green) in hepatocyte and in cancer cell, note that DLC2 immunoreactivity forms large granules in hepatocyte, whereas in cancer cell DLC2 immunoreactivity is scattered along cellular cytoplasm. Scale bars: a,c,d,f,g) $50 \mu \mathrm{m}$; e) $20 \mu \mathrm{m}$; b,j,k) 100 $\mu \mathrm{m} ; 1, \mathrm{~m}) 5 \mu \mathrm{m}$. 


\section{Discussion}

In the current study we document the presence of both protein and gene $D L C 2$ in two types of hepatocellular carcinoma classical and fibrolamellar one. We demonstrate novel results which indicate that $D L C 2$ unlike its relative, $D L C 1$, does not undergo genetic deletion in cancer cells. Moreover, we display that the DLC2 protein is more abundantly expressed in cancer cells when compared to normal hepatocytes, especially when the cancer develops with the considerable inflammation background. We show also that the tissues with macroregenerative nodules have a similar level of DLC2 immunoreactivity to the level observed in normal liver sections. For both the gene and the DLC2 protein status we did not observe any differences between the two types of hepatocellular carcinoma classical and fibrolamellar variant. It was found that the expression and function of the DLC2 gene in cancer is ambiguous. Early reports have shown that DLC2 possesses growth suppressive function and its expression is reduced in about $18 \%$ of hepatocellular carcinoma tissues. ${ }^{21}$ Subsequent studies revealed tumor suppressive function of DLC2 through RhoA downregulation followed by inhibition of cell proliferation and migration in vitro. ${ }^{20}$ In a cancer profiling study, which included other types of solid tumors, i.e. lung, breast and rectal, Ullmannova et al. showed that both DLC1 and DLC2 are underexpressed, however to a different degree. ${ }^{19}$ On contrast to the studies mentioned above, Yau et al. demonstrated, using the knockout mouse models, that DLC2 deficiency did not predispose to HCC formation, even in the presence of chemical hepatocarcinogen. ${ }^{23}$ Interesting results were also presented in a work of
Basak et al., where authors showed that in the mammary cancer model the $D L C 2$ deletion did not change the tumor growth, however it had an impact on the metastasis formation, which suggests its metastasis suppressor rather than tumor suppressor role. ${ }^{24}$ Our results did not confirm the DLC2 deficiency as a common feature of HCC at the protein and gene levels in our experimental group. Similar results were presented in the work of Wang et al., where authors showed that the major member of the DLC family whose expression is reduced in $\mathrm{HCC}$ is DLC1, while the expression of DLC2 was not diminished and even was increased in the subset of tumors. ${ }^{25}$ In our experimental group we noted increased DLC2 immunoreactivity in a small part of HCC samples. Such increased immunoreactivity was associated with the abundant presence of inflammatory cells. It appears that the inflammation process may affect the expression of DLC2, but the mechanism of this relationship remains to be determined.

Interestingly, we found DLC2 immunoreactivity in macroregenerative nodules to be similar to the one seen in the control group. These structures represent the state in the liver where active tissue remodeling occurs as a result of different liver injury. It looks that the abundant presence of DLC2 is not common for such benign lesions, and some additional factors in cancer cells trigger its excessive expression. Such result indicates that DLC2 immunoreactivity can potentially differentiate the non-malignant nodules vs HCC. However, such use of DLC2 immunostaining for diagnostic purposes requires further research.

The loss of one copy of the gene was established as the principal mechanism of DLC1 underexpression in $\mathrm{HCC}$, while the status of the $D L C 2$ gene was studied less extensively. In our study we detected a loss of gene copy in a small percentage of the tumor samples tested. Available literature data indicated that loss of $D L C 2$ copy number occurs only in a subset of HCC specimens which implicates that such a mechanism of the protein underrepresentation is not a common attribute in this type of cancer. $^{25}$

We observed DLC2 protein localized mainly in the cytoplasm of cancer cells. As was demonstrated on hepatoma cells, DLC2 was found to be located in mitochondria through the START domain. ${ }^{26}$ To investigate this issue on HCC sections, we performed double immunofluorescence studies with anti-DLC2 and anti-mitochondrial antigens. However, we did not find a significant colocalization of both proteins, in both normal hepatocytes and cancer cells, indicating that this interaction does not appear to be a common feature of hepatocellular carcinoma cells. Interestingly, we found a cancer cell population with DLC2 located in nuclei, which was not observed in normal hepatocytes. So far there were no studies showing DLC2 localized in cancer cell nuclei, but the presence of the SAM domain within the protein structure, responsible i.e. for the protein - DNA/RNA interaction suggests possible DLC2 interactions with chromatin structures and thus possible impact on various nuclear processes. ${ }^{27}$

DLC2 belongs to the broader family of DLC proteins with high structure homology. All DLC proteins contain three functional domains: SAM, RhoGAP and START domain. ${ }^{12}$ Since DLC2 structure is $50 \%$ identical to DLC1 their function may be overlapping. Therefore, DLC2 presence in cancer cells should be taken into consideration whenever other members of this family,

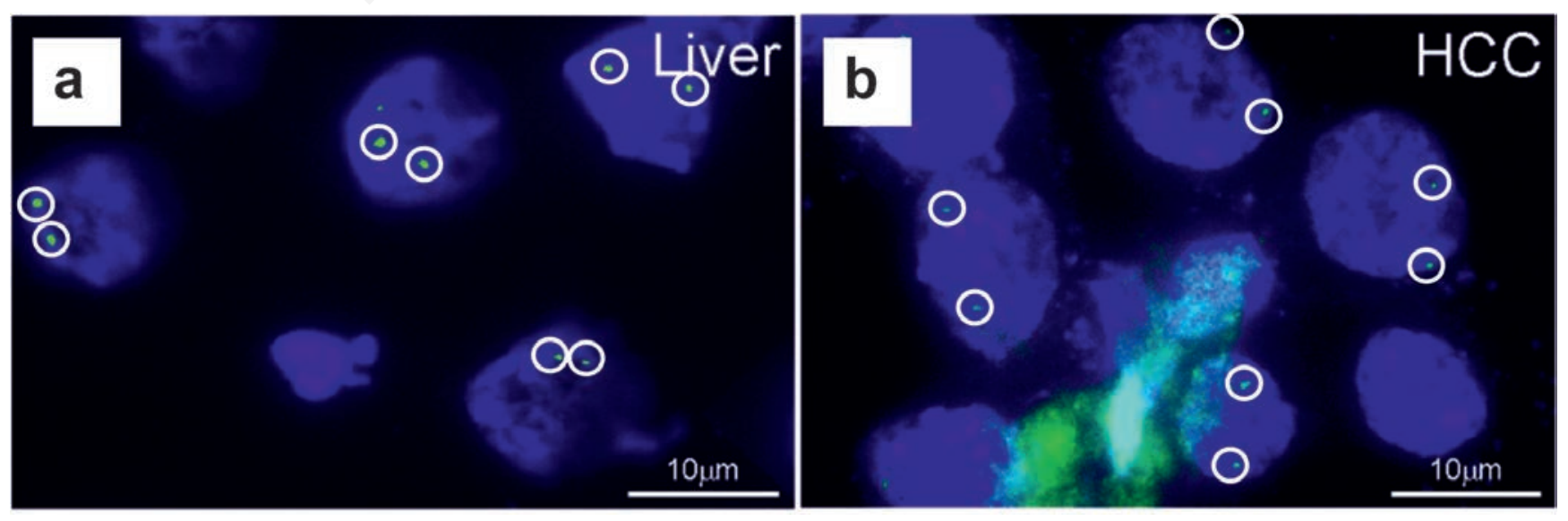

Figure 2. Fluorescence in situ hybridization with $D L C 2$ probe in the normal liver and hepatocellular carcinoma. a) FISH result with $D L C 2$ probe in the normal liver. b) FISH result with $D L C 2$ probe in HCC specimen. Probe signals were marked with round circles. 
especially DLC1 are considered for possible use in prevention, diagnosis, and therapies.

To summarize, we demonstrated that DLC2 protein expression is present in $\mathrm{HCC}$ sections. Our findings question the DLC2 tumor suppressor activity by the mechanism of gene and protein depletion in this type of cancer. Increased DLC2 expression associated with the inflammation suggests its possible association with the inflammatory process. A more detailed analysis of the mechanisms regulating the activity of DLC2 in the normal development and pathogenesis of cancer is required to explain its participation in the onset of HCC.

\section{References}

1. Siegel RL, Miller KD, Jemal A. Cancer statistics, 2018. CA Cancer J Clin 2018; 68:7-30.

2. Bray F, Ferlay J, Soerjomataram I, Siegel RL, Torre LA, Jemal A. Global cancer statistics 2018: GLOBOCAN estimates of incidence and mortality worldwide for 36 cancers in 185 countries. CA Cancer J Clin 2018;68:394424.

3. Ghouri YA, Mian I, Rowe JH. Review of hepatocellular carcinoma: Epidemiology, etiology, and carcinogenesis. J Carcinog 2017;16:1.

4. Llovet JM, Montal R, Sia D, Finn RS. Molecular therapies and precision medicine for hepatocellular carcinoma. Nat Rev Clin Oncol 2018;15:599-616.

5. Llovet JM, Zucman-Rossi J, Pikarsky E, Sangro B, Schwartz M, Sherman M, et al. Hepatocellular carcinoma. Nat Rev Dis Primers 2016;2:16018.

6. Kakar S, Burgart LJ, Batts KP, Garcia J, Jain D, Ferrell LD. Clinicopathologic features and survival in fibrolamellar carcinoma: comparison with conventional hepatocellular carcinoma with and without cirrhosis. Mod Pathol 2005;18:1417-23.

7. Lalazar G, Simon SM. Fibrolamellar carcinoma: Recent advances and unre- solved questions on the molecular mechanisms. Semin Liver Dis 2018; 38:51-9.

8. Graham RP. Fibrolamellar carcinoma: What is new and why it matters. Surg Pathol Clin 2018;11:377-87.

9. Graham RP, Torbenson MS. Fibrolamellar carcinoma: A histologically unique tumor with unique molecular findings. Semin Diagn Pathol 2017; 34:146-52.

10. Ellenbroek SI, Collard JG. Rho GTPases: functions and association with cancer. Clin Exp Metastasis 2007;24:657-72.

11. Heasman SJ, Ridley AJ. Mammalian Rho GTPases: new insights into their functions from in vivo studies. Nat Rev Mol Cell Biol 2008;9:690-701.

12. Braun AC, Olayioye MA. Rho regulation: DLC proteins in space and time. Cell Signal 2015;27:1643-51.

13. Wong CM, Yam JW, Ching YP, Yau TO, Leung TH, Jin DY, et al. Rho GTPaseactivating protein deleted in liver cancer suppresses cell proliferation and invasion in hepatocellular carcinoma. Cancer Res 2005;65:8861-8.

14. Healy KD, Hodgson L, Kim TY, Shutes A, Maddileti S, Juliano RL, et al. DLC1 suppresses non-small cell lung cancer growth and invasion by RhoGAPdependent and independent mechanisms. Mol Carcinog 2008;47:326-37.

15. Liu X, Pan YJ, Zheng JN, Pei DS. The role of tumor suppressor DLC-1: Far from clear. Anticancer Agents Med Chem 2017;17:896-901.

16. Song LJ, Liu Q, Meng XR, Li Sh L, Wang LX, Fan QX, et al. DLC-1 is an independent prognostic marker and potential therapeutic target in hepatocellular cancer. Diagn Pathol 2016;11:19.

17. Du J, Bai F, Zhao P, Li X, Gao L, Ma C, et al. Hepatitis B core protein promotes liver cancer metastasis through miR382-5p/DLC-1 axis. Biochim Biophys Acta Mol Cell Res 2018;1865:1-11.

18. Struski S, Doco-Fenzy M, CornilletLefebvre P. Compilation of published comparative genomic hybridization studies. Cancer Genet Cytogenet 2002;135:63-90.

19. Ullmannova V, Popescu NC. Expression profile of the tumor suppressor genes DLC-1 and DLC-2 in solid tumors. Int J Oncol 2006;29:112732.

20. Leung TH, Ching YP, Yam JW, Wong CM, Yau TO, Jin DY, et al. Deleted in liver cancer 2 (DLC2) suppresses cell transformation by means of inhibition of RhoA activity. Proc Natl Acad Sci USA 2005;102:15207-12.

21. Ching YP, Wong CM, Chan SF, Leung $\mathrm{TH}, \mathrm{Ng} \mathrm{DC}$, Jin DY, et al. Deleted in liver cancer (DLC) 2 encodes a RhoGAP protein with growth suppressor function and is underexpressed in hepatocellular carcinoma. J Biol Chem 2003;278:10824-30.

22. Cremer M, Grasser F, Lanctot C, Muller $\mathrm{S}$, Neusser M, Zinner R, et al. Multicolor 3D fluorescence in situ hybridization for imaging interphase chromosomes. Methods Mol Biol 2008;463:205-39.

23. Yau TO, Leung TH, Lam S, Cheung OF, Tung EK, Khong PL, et al. Deleted in liver cancer 2 (DLC2) was dispensable for development and its deficiency did not aggravate hepatocarcinogenesis. PLoS one 2009;4:e6566.

24. Basak P, Leslie H, Dillon RL, Muller WJ, Raouf A, Mowat MRA. In vivo evidence supporting a metastasis suppressor role for Stard13 (Dlc2) in ErbB2 (Neu) oncogene induced mouse mammary tumors. Genes Chromosomes Cancer 2018;57:182-91.

25. Wang D, Qian X, Rajaram M, Durkin ME, Lowy DR. DLC1 is the principal biologically-relevant down-regulated DLC family member in several cancers. Oncotarget 2016;7:45144-57.

26. Ng DC, Chan SF, Kok KH, Yam JW, Ching YP, Ng IO, et al. Mitochondrial targeting of growth suppressor protein DLC2 through the START domain. FEBS Lett 2006;580:191-8.

27. Qiao F, Bowie JU. The many faces of SAM. Sci STKE 2005;2005:re7. 